\title{
Effects of concurrent exercise training on muscle dysfunction and systemic oxidative stress in older people with COPD
}

\author{
Julian Alcazar $^{1,2}$ (D) | Jose Losa-Reyna ${ }^{1,2,3} \quad$ | Carlos Rodriguez-Lopez ${ }^{1,2}$ | Roberto Navarro- \\ Cruz $^{1,2}$ | Ana Alfaro-Acha ${ }^{2,3}$ ｜ Ignacio Ara ${ }^{1,2}$ | Francisco J. García-García ${ }^{2,3}$ | \\ Luis M. Alegre ${ }^{1,2}$ (D) | Amelia Guadalupe-Grau ${ }^{2,3,4}$ (D)
}

${ }^{1}$ GENUD Toledo Research

Group, Universidad de Castilla-La Mancha,

Toledo, Spain

${ }^{2}$ CIBER of Frailty and Healthy Aging (CIBERFES), Madrid, Spain

${ }^{3}$ Hospital Virgen del Valle, Complejo Hospitalario de Toledo, Toledo, Spain

${ }^{4}$ ImFINE Research Group, Universidad Politécnica de Madrid, Madrid, Spain

\section{Correspondence}

Luis M. Alegre, GENUD Toledo Research Group, Universidad de Castilla-La Mancha, Avda. Carlos III, S/N, 45071, Toledo,

Spain.

Email: luis.alegre@uclm.es

Amelia Guadalupe-Grau, ImFINE Research Group, Universidad Politécnica de Madrid, c/ Martín Fierro 7, s/n, Madrid, Spain.

Email: amelia.guadalupe@upm.es

\section{Funding information}

This work was supported by the Ministerio de Economía y Competitividad of the Government of Spain (DEP2015-69386-R and BES-2016-077199) (MINECO/ FEDER, EU); the Biomedical Research Networking Center on Frailty and Healthy Aging (CIBERFES) and FEDER funds from the European Union (CB16/10/00477 and CB16/10/00456); the Ministerio de Educación, Cultura y Deporte of the Government of Spain (FPU014/05106); and the Universidad de Castilla-La Mancha (2016/11635).
Oxidative stress is associated with disease severity and limb muscle dysfunction in COPD. Our main goal was to assess the effects of exercise training on systemic oxidative stress and limb muscle dysfunction in older people with COPD. Twenty-nine outpatients with COPD (66-90 years) were randomly assigned to a 12-week exercise training (ET; high-intensity interval training (HIIT) plus power training) or a control (CT; usual care) group. We evaluated mid-thigh muscle cross-sectional area (CSA; computed tomography); vastus lateralis (VL) muscle thickness, pennation angle, and fascicle length (ultrasonography); peak $\mathrm{VO}_{2}$ uptake $\left(\mathrm{VO}_{2 \text { peak }}\right)$ and work rate $\left(\mathrm{W}_{\text {peak }}\right)$ (incremental cardiopulmonary exercise test); rate of force development (RFD); maximal muscle power $\left(\mathrm{P}_{\max }\right.$; force-velocity testing); systemic oxidative stress (plasma protein carbonylation); and physical performance and quality of life. ET subjects experienced changes in mid-thigh muscle CSA $(+4 \%)$, VL muscle thickness $(+11 \%)$ and pennation angle $(+19 \%), \mathrm{VO}_{2 \text { peak }}(+14 \%), \mathrm{W}_{\text {peak }}(+37 \%), \mathrm{RFD}(+32 \%$ to $65 \%)$, $\mathrm{P}_{\max }(+38 \%$ to $51 \%)$, sit-to-stand time $(-24 \%)$, and self-reported health status $(+20 \%)($ all $P<0.05)$. No changes were noted in the CT group $(P>0.05)$. Protein carbonylation decreased among ET subjects $(-27 \% ; P<0.05)$, but not in the CT group $(P>0.05)$. Changes in protein carbonylation were associated with changes in muscle size and pennation angle ( $r=-0.44$ to -0.57$)$, exercise capacity $(r=-0.46)$, muscle strength $(r=-0.45)$, and sit-to-stand performance $(r=0.60)$ (all $P<0.05)$. The combination of HIIT and power training improved systemic oxidative stress and limb muscle dysfunction in older people with COPD. Changes in oxidative stress were associated with exercise-induced structural and functional adaptations.

\section{K E Y W O R D S}

aerobic capacity, endurance training, lung disease, muscle architecture, power output, protein carbonyls, redox status, resistance training 


\section{INTRODUCTION}

Chronic obstructive pulmonary disease (COPD) is a chronic lung disease characterized by airway and alveolar abnormalities that lead to persistent respiratory symptoms and airflow limitation that are not fully reversible. ${ }^{1}$ The most characteristic symptom of COPD is chronic and progressive dyspnea, but several systemic manifestations and consequences of COPD (eg, cardiovascular disease, limb muscle dysfunction and depression) have been reported to produce a further worsening in quality of life and increased mortality risk. A major and highly prevalent manifestation of COPD is limb muscle dysfunction, which is associated with poor quality of life and increased mortality. ${ }^{2}$ Limb muscle dysfunction is characterized by a decreased proportion of type I muscle fibers and oxidative capacity, and reduced muscle cross-sectional area, strength, and endurance. ${ }^{2}$ A likely contributor to the progression of the disease and its systemic effects is oxidative stress, which results from the imbalance between the generation of reactive oxygen and nitrogen species (RONS) and the efficiency of antioxidant mechanisms. ${ }^{3,4}$ It is generally accepted that patients with COPD present increased levels of protein carbonylation, which is the most abundant by-product of oxidative damage to proteins. ${ }^{5}$ Protein carbonylation can modify protein function, disrupting normal cell function, and physiologic mechanisms, ${ }^{6}$ and has been associated with COPD severity. ${ }^{7}$ In addition, protein carbonylation has been found to be negatively associated with skeletal muscle mass, ${ }^{8,9}$ muscle strength, and exercise tolerance in COPD. ${ }^{10,11}$

Pulmonary rehabilitation is well known to improve limb muscle dysfunction, exercise capacity, and quality of life in patients with COPD. ${ }^{12}$ Exercise training is regarded as the cornerstone of pulmonary rehabilitation, ${ }^{13}$ and researchers are encouraged to focus on further factors that remain uncertain, such as finding the optimal exercise parameters to maximize the benefits of pulmonary rehabilitation in COPD. ${ }^{12}$ Both continuous and interval endurance training have been reported to be effective to improve dyspnea, exercise capacity, and quality of life in patients with COPD. ${ }^{14}$ In addition, the combination of endurance and resistance training has been shown to provide greater adaptations in muscle strength and quality of life compared with endurance training alone. ${ }^{15}$ Power training has been proven to achieve greater functional benefits than traditional resistance training in older people, ${ }^{16}$ and it might be especially beneficial to patients with COPD because of the lower cardiovascular loading ${ }^{17}$ and higher muscle oxygenation levels ${ }^{18}$ that are experienced during exercise compared with traditional strength training. In addition, muscle power has recently been found to be reduced by $30 \%$ in older people with COPD when compared with their healthy counterparts. ${ }^{19}$ However, to date no studies have investigated the effects of power training in older people with COPD.
Exercise training has also been shown to provide chronic benefits in oxidative stress among healthy individuals; however, several studies have failed to show a reduction in oxidative stress among patients with COPD after an exercise training intervention. ${ }^{11,20-25}$ All these studies implemented endurance training alone, and thus, the effects of an exercise program combining high-intensity interval training (HIIT) and power training on oxidative stress among patients with COPD remain to be elucidated. The main goal of the present investigation was to evaluate the effect of an exercise training program combining HIIT and power training on limb muscle dysfunction, exercise tolerance, physical performance, quality of life, and systemic oxidative stress in older people with COPD.

\section{MATERIAL AND METHODS}

\section{1 | Study design}

The study was designed as a randomized controlled parallel two-group study. The participants were randomly assigned to one of the two groups: exercise training (ET) or control (CT). Stratified randomization was used to achieve approximate balance regarding physical function (SPPB $<10$ and SPPB $\geq 10$ ) and sex (women and men). The subjects in the ET group participated in a 12-week exercise training program combining HIIT and power training, while the CT group received no intervention (ie, usual care) during the same time period. Before and after the 12-week exercise training program, both groups visited the laboratory several times over 2 weeks in order to familiarize themselves with the procedures and equipment (before), and for the evaluation of various outcomes (before and after).

\subsection{Study population}

Prior to entering the study, the subjects had to be $\geq 65$ years old, diagnosed with COPD by a pulmonologist and be clinically stable. Exclusion criteria included having participated in an endurance and/or resistance training program within the previous 12 months, a short physical performance battery (SPPB) score $<4$, severe cognitive impairment (mini-mental state examination (MMSE) score $<20$ ), neuromuscular or joint injury, stroke, myocardial infarction or bone fracture in the previous 12 months, uncontrolled hypertension (>200/110 mm Hg), or terminal illness. COPD severity was evaluated by the BODE (body mass index, obstruction, dyspnea, and exercise) index. ${ }^{26}$ Finally, twenty-nine outpatients with COPD participated in this investigation and were randomized into two groups (Table 1). All the subjects provided written informed consent. The study was performed in accordance with the Helsinki Declaration and approved by the Ethical Committee of the Toledo Hospital. 


\section{3 | Physical performance and quality of life}

Physical performance was assessed by the SPPB score, which evaluates static balance, 4-m habitual gait speed, and five-repetition sit-to-stand (STS) time. Exercise capacity was assessed by the 6-minute walking distance (6-MWD). Healthrelated quality of life was assessed using the EQ-5D-5L questionnaire, consisting of two parts: the first part evaluates the prevalence of problems in five dimensions (mobility, selfcare, usual activities, pain/discomfort, and anxiety/depression) obtaining an EQ-index (0-1), and in the second part, the EQ visual analogue scale (EQ-VAS) records self-reported health status (0-100).

\subsection{Anthropometrics and mid-thigh composition}

A stadiometer and scale device (Seca 711, Seca, Hamburg, Germany) was used to record the height (m), body mass ( $\mathrm{kg}$ ), and body mass index (BMI; $\mathrm{kg} \mathrm{m}^{-2}$ ) of the participants.

Mid-thigh composition was measured using computed tomography (BrightSpeed, GE Healthcare). The scan was performed at the midpoint of the distance between the superior border of the greater trochanter and the inferior border of the lateral epicondyle of the right leg. Ten 10-mm-thick axial images (120 kVp, 200-250 mAs) were obtained and saved as DICOM images. Skeletal muscle tissue was identified as those pixels between 0 and 100 Hounsfield units (HU), and

TA B L E 1 Main characteristics of the study participants

\begin{tabular}{|c|c|c|}
\hline & ET group & CT group \\
\hline & Mean \pm SD & Mean \pm SD \\
\hline $\operatorname{Sex}(\mathrm{F} / \mathrm{M})$ & $3 / 11$ & $2 / 13$ \\
\hline Age (y) & $77.7 \pm 7.9$ & $79.8 \pm 6.4$ \\
\hline $\operatorname{BMI}\left(\mathrm{kg} \mathrm{m}^{-2}\right)$ & $28.8 \pm 3.0$ & $32.5 \pm 5.9$ \\
\hline MMSE score & $24.1 \pm 3.5$ & $25.9 \pm 3.3$ \\
\hline SPPB score & $10.0 \pm 2.4$ & $9.9 \pm 2.5$ \\
\hline BODE index & $3.1 \pm 1.7$ & $2.7 \pm 2.1$ \\
\hline $\mathrm{FEV}_{1}(\%$ pred $)$ & $47.4 \pm 18.1$ & $58.7 \pm 15.2$ \\
\hline FVC (\% pred) & $72.8 \pm 21.6$ & $82.1 \pm 24.4$ \\
\hline $\mathrm{FEV}_{1} / \mathrm{FVC}(\%)$ & $50.0 \pm 16.6$ & $55.1 \pm 10.7$ \\
\hline $\mathrm{SpO}_{2}(\%)$ & $90.7 \pm 2.9$ & $93.0 \pm 2.9$ \\
\hline 6-MWD (m) & $420.3 \pm 111.6$ & $400.1 \pm 117.5$ \\
\hline
\end{tabular}

Note: There were no significant differences between groups for any of the variables.

Abbreviations: 6-MWD, 6-min walking distance; BMI, body mass index; CT, control; ET, exercise training; F, female; FEV1, forced expiratory volume in one second; FVC, forced vital capacity; M, male; MMSE, mini-mental state examination; $\mathrm{SpO}_{2}$, peripheral capillary oxygen saturation; $\mathrm{SPPB}$, short physical performance battery. fat tissue as those between -30 and $-190 \mathrm{HU}$. Muscle crosssectional area (CSA) and mean attenuation values, and fat CSA were measured by outlining the borders of the desired tissues while avoiding nerves and vessels.

Vastus lateralis (VL) muscle thickness and muscle architecture were assessed using B-mode ultrasonography (MyLab 25, EsaoteBiomedica) with a $50 \mathrm{~mm}, 10-15 \mathrm{MHz}$ linear-array probe. Resting ultrasound images were taken on the same marks realized for the CT scan with the participants lying on their back and the knee resting slightly flexed at $150^{\circ}\left(180^{\circ}=\right.$ full knee extension $)$. The transducer was aligned in the fascicle plane to be able to visualize an optimal portion of fascicles on the ultrasound screen. Muscle thickness was measured as the average of the perpendicular distance between the superficial and deep aponeuroses of the VL muscle at three different points on the image (left border, midpoint, and right border). VL pennation angle and fascicle length were measured from the visible portion of two fascicles within the same image. Linear extrapolation of fibers and aponeuroses was used when a small portion of the fascicle extended off the ultrasound window. Consistent selection of measurement sites across testing days was verified by recording the probe positions onto transparent acetate paper and using easily identifiable infiltrations of fatty and connective tissues on the sonograms as landmarks. ${ }^{27}$ Both CT and ultrasound images were digitally analyzed (ImageJ $1.51 \mathrm{j} 8$, $\mathrm{NIH})$ by the same operator.

\section{5 | Resting peripheral capillary oxygen saturation and lung function}

After a 10-minute rest, peripheral capillary oxygen saturation $\left(\mathrm{SpO}_{2}\right)$ was recorded in the sitting position with a pulse oximetry device (CMS50F, Contec Medical S). Then, all participants underwent flow volume tests using a spirometer (Spirosoft, GE Healthcare). The measurements included the recording of $\mathrm{FEV}_{1}, \mathrm{FVC}$, and $\mathrm{FEV}_{1} / \mathrm{FVC}$ values, with the highest value from at least three properly performed measurements being used for analysis.

\subsection{Cardiopulmonary exercise testing}

A progressive incremental exercise protocol on an electromagnetically braked cycle ergometer (800S, Ergoline, Bitz, Germany) was conducted at a constant pedal speed of 60-80 rpm. After a 3-minute rest and a 3-minute warm-up at $25 \mathrm{~W}$, exercise work rate was increased by $10 \mathrm{~W}$ every min until voluntary cessation of exercise or until subjects were unable to maintain pedaling frequency (ie, $<60 \mathrm{rpm}$ ). Peak work rate $\left(\mathrm{W}_{\text {peak }}\right)$ was defined as the highest work level reached and maintained at a pedaling frequency of $\geq 60 \mathrm{rpm}$ for 30 seconds. Ventilation and pulmonary gas exchange were obtained breath-by-breath through a tightly sealing 
breathing mask attached to a digital volume flow sensor and an extremely fast $\mathrm{O}_{2}$ and $\mathrm{CO}_{2}$ gas analyser (Oxycon Pro). Heart rate was registered using a heart rate monitor (A300, Polar Electro Oy) and peripheral capillary oxygen saturation $\left(\mathrm{SpO}_{2}\right)$ with a pulse oximetry device (CMS50F, Contec Medical S). Offline data analysis was carried out for determination of maximal exercise capacity using peak pulmonary oxygen uptake $\left(\mathrm{VO}_{2 \text { peak }}\right.$; highest volume of oxygen uptake averaged over a 20 -second period).

\section{7 | Maximal voluntary isometric contraction}

Maximal isometric force (MIF) and rate of force development (RFD) were measured during a maximal voluntary isometric contraction in the leg press exercise (Element+, Technogym, Italy). A force plate (Type 9286BA) was installed on the leg press apparatus in order to capture force values. The subjects sat in the upright position, with their knees flexed at $90^{\circ}\left(180^{\circ}=\right.$ full knee extension $)$, feet always placed in the same position relative to the surface of the force platform, and their arms at their sides with their hands holding onto the handles of the apparatus. Two chains were attached between the seat and the platform of the leg press and tensed as much as possible in order to impede any movement during the isometric testing and make the system as rigid as possible. The subjects were instructed to push with their legs on the force platform as fast and hard as possible after the cue "ready, set, go!" for at least 4 seconds. Strong verbal encouragement and visual feedback during and after each contraction, respectively, were provided to ensure maximal performance. The subjects performed several consecutive maximal isometric contractions (separated by 60 seconds) until 5 acceptable trials (stable baseline force and no countermovement immediately prior to the onset of the contraction) were obtained. External forces were collected at a sampling rate of $1000 \mathrm{~Hz}$ and processed with specific software (BioWare 5.3.0.7). An automated threshold method was used to detect contraction onset as the instant when force rose above three standard deviations the baseline force noise $(2.4 \pm 0.6 \mathrm{~N})$. MIF and RFD at 100, 200, and $400 \mathrm{~ms}$ were obtained from the average of the two best trials in terms of MIF and used for further analysis. $\mathrm{CV}$ values for these measures were found to be $4.9 \pm 3.4 \% ; 14.9 \pm 11.1 \%, 10.7 \pm 9.3 \%$, and $7.5 \pm 7.6 \%$, respectively. In addition, the time needed to reach 0.5 body weight (BW) was calculated as a functional measure of rapid isometric force production.

\section{8 | Force-velocity relationship}

A detailed description and validation of the F-V and muscle power testing has been previously reported. ${ }^{28}$ The subjects performed two sets of three repetitions with increasing loads
(5-10 kg increments) in the leg press and chest press exercises (Element+, Technogym) until the one repetition maximum (1RM) was achieved. Mean force and velocity data during the concentric phase of each repetition were recorded by a linear position transducer device (T-Force System, Ergotech, Spain). Repetitions were performed as fast and strongly as possible. The highest mean velocity for each load was plotted on a Microsoft Excel® custom-made template, ${ }^{28}$ and a linear regression equation was fitted simultaneously during the F-V evaluation. Several variables were extracted from the F-V regression equation as previously reported ${ }^{28}$ : force-intercept or maximal force $\left(\mathrm{F}_{0}\right)$, velocity-intercept or maximal velocity $\left(\mathrm{V}_{0}\right)$, maximal muscle power $\left(\mathrm{P}_{\max }\right)$, and optimal load (ie, the load yielding $\left.\mathrm{P}_{\max }\right)$. CV values have been reported to be $2.6 \%-5.6 \%$ for these variables. ${ }^{28}$

\section{9 | Protein carbonylation}

Blood samples were obtained at rest from an antecubital vein after a $\geq 12$-hour overnight fast in tubes containing ethylenediaminetetraacetic acid (EDTA) (BD Vacutainer, Stockholm, Sweden), and 72-96 hours before and after the last training session. EDTA-containing tubes were immediately centrifuged $\left(1200 \mathrm{~g}, 10\right.$ minutes, $\left.4^{\circ} \mathrm{C}\right)$, and plasma was put into $500-\mu \mathrm{L}$ aliquots and stored at $-80^{\circ} \mathrm{C}$ until analysis. Total protein content in plasma samples was quantified using the bicinchoninic acid assay. The presence of protein carbonyl groups was assessed using the Oxyblot Protein Oxidation Detection Kit (Millipore) according to the manufacturer's protocol. The carbonyl groups in the protein side chains were derivatized to 2,4-dinitrophenilhydrazone (DNP-hydrazone) by reaction with 2,4-dinitrophenylhydrazine (DNPH). The DNP-derivatized protein samples $(18 \mu \mathrm{g})$ were loaded onto a polyacrylamide gel (Criterion TGX Stain-Free 4\%-20\%, Bio-Rad, Denmark). In addition, a control sample was loaded onto three walls of each gel in order to account for the variability between gels. Then, the DNP-derivatized protein samples were separated during SDS-electrophoresis; the trihalo compounds contained in the gels were activated using UV light exposure for 1 minute followed by a 1-second exposure image (Chemidoc XRS + system, Bio-Rad, Denmark); and the activated gel was then electrophoretically transferred to a polyvinylidene difluoride (PVDF) membrane (Immun-Blot Low Fluorescence PVDF Membrane, Bio-rad, Denmark). PVDF membranes were imaged after transfer in order to normalize the results for lane protein. After that, the membranes were blocked with 5\% skim milk in phosphate-buffered saline containing $0.1 \%$ Tween 20 (PBS-T) and incubated overnight at $4{ }^{\circ} \mathrm{C}$ with the corresponding primary antibody (rabbit anti-DNP antibody, ref. 90451, Millipore) (diluted 1:150 into blocking buffer). The membranes were then incubated with the secondary antibody (goat anti-rabbit $\operatorname{IgG}$ HRP-conjugated, ref. 90452, Millipore) (diluted 1:300 into 
blocking buffer) and the protein bands visualized using the adequate reagents (Clarity Western ECL Substrate, Bio-Rad, Denmark) and the Chemidoc XRS + system. The abundance of protein carbonylation was assessed by densitometry of each lane, carefully ensuring avoidance of pixel saturation (Image Lab 6.0.0, Bio-rad, Denmark). The results were normalized to the total protein lane and further to the control sample.

\subsection{0 | Exercise training program}

The participants assigned to the ET group attended 24 exercise training sessions on non-consecutive days during 12 weeks (ie, 2 days a week). Each session consisted of a 5-minute warm-up on the cycle ergometer (60-80 rpm; $40 \%$ of $\left.\mathrm{W}_{\text {peak }}\right)$, followed by power training in the leg press and chest press exercise machines, and endurance HIIT on the cycle ergometer. Exercise volume and intensity were carefully periodized during the 12-week training period (Supplementary Material). Briefly, for the power training section of the exercise program, the subjects performed 2-3 sets of 8-12 repetitions at $50 \%-60 \%$ of 1RM for each reported exercise during the first 3 weeks. Then, from week 4 to 12 the subjects performed 3 sets of 8 repetitions with the optimal load (ie, the load yielding the maximal muscle power during the F-V test). The F-V relationship was evaluated every 4 weeks to adjust the training loads according to the progress made by the subjects. The subjects were asked to perform the concentric phase of each repetition as fast and strongly as possible, while no instructions were given for the eccentric phase. During the HIIT section of the exercise program, the subjects performed five sets of 90 seconds at a light-moderate intensity (active recovery: $40 \%$ of $\mathrm{W}_{\text {peak }}$ ) plus 30 seconds at a heavy intensity ( $80 \%$ of $\mathrm{W}_{\text {peak }}$ ) on the cycle ergometer during the first 3 weeks. From week 4 to 12 , the number of sets was incremented to 10, with the intensity also being augmented in certain weeks during the exercise program. In order to adjust the exercise intensity to the progress made by the subjects, $\mathrm{W}_{\text {peak }}$ was determined again during the sixth week of training. Strong verbal encouragement was given to all the subjects while performing the exercises. All the training sessions were supervised

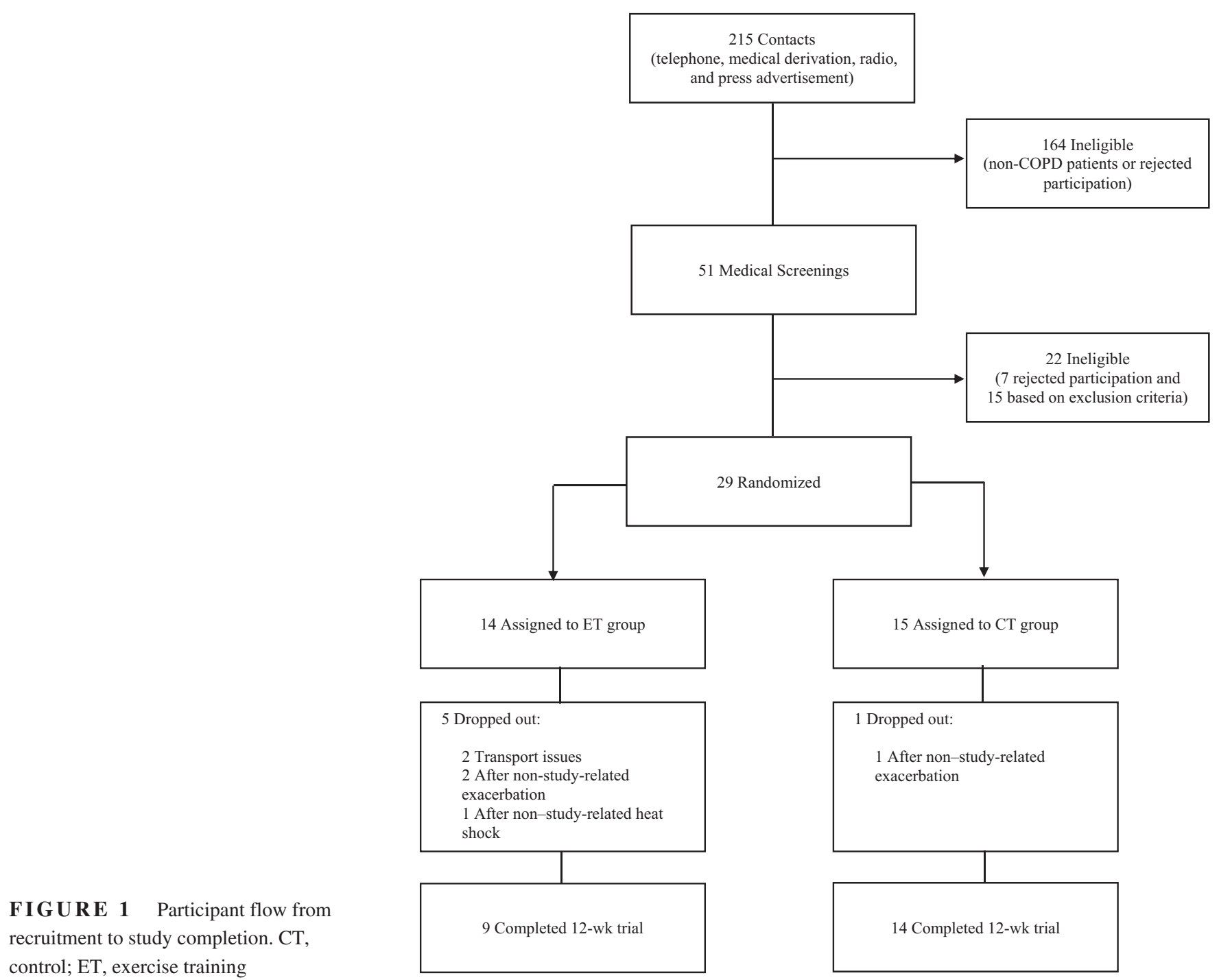


interaction for $\mathrm{VO}_{2 \text { peak }}$ and $\mathrm{W}_{\text {peak }}$ values $(P<0.05)$. The ET group increased $\mathrm{VO}_{2 \text { peak }}(+14.1 \% ; P<0.05)$ and $\mathrm{W}_{\text {peak }}$ $(+36.9 \% ; P<0.01)$, with no changes in the $\mathrm{CT}$ group $(-0.6 \%$ and $+2.9 \%$, respectively; $P>0.05)$. By contrast, no significant time $\times$ group interactions were observed in resting lung function variables $(P>0.05)$, except for FEV1/FVC values, for which the CT group showed an increase $(+7.7 \% ; P<0.05)$, and no changes were noted in the ET group $(-5.7 \% ; P>0.05)$.

\section{4 | Muscle strength and power}

A significant time $\times$ group interaction was observed in MIF, although no significant changes were found in any of the groups (ET: $+10.4 \%$ vs CT: $-10.0 \%$; both $P>0.05$ ) (Table $3)$. A significant time $\times$ group interaction was also reported regarding RFD values at 100,200 , and $400 \mathrm{~ms}(P<0.01)$. The ET group improved RFD at 100, 200, and $400 \mathrm{~ms}$ by $65.2 \%, 48.8 \%$, and $31.7 \%$, respectively (all $P<0.05$ ), while no changes were reported in the CT group (all $P>0.05$ ). In addition, a significant time $\times$ group interaction was found in time to reach $0.5 \mathrm{BW}$, with ET subjects tending to reduce it
$(-39.3 \% ; P=0.075)$ and the $\mathrm{CT}$ group showing no changes $(+2.5 \% ; P>0.05)$. On the other hand, an overall positive effect of the exercise program was observed in the F-V relationship of the participants. Significant time $\times$ group interactions were observed for both leg press (Figure 4A) and chest press (Figure 4B) $1 \mathrm{RM}, \mathrm{V}_{0}$, and $\mathrm{P}_{\max }$ values (all $P<0.05$ ), but not for $\mathrm{F}_{0}(P=0.062$ and 0.066 , respectively). Increments in $1 \mathrm{RM}(+19.6 \%$ and $+28.6 \%), \mathrm{V}_{0}(+40.7 \%$ and $+15.7 \%)$, and $\mathrm{P}_{\max }(+51.2 \%$ and $+37.8 \%)$ were reported in ET subjects in the leg press and chest press exercises, respectively (all $P<0.05$ ). No differences in leg press and chest press outcomes were observed in the $\mathrm{CT}$ group after the 12-week period $(P>0.05)$.

\section{5 | Physical performance and quality of life}

A significant time $\times$ group interaction was noted in habitual gait speed and STS performance (both $P<0.05$ ) (Table 3). Habitual gait speed and STS time were improved by $8.2 \%$ and $24.3 \%$, respectively, in the ET subjects, while both performances declined by $5.0 \%$ and $15.8 \%$, respectively, among
TA B L E 2 Effects of exercise training on cardiopulmonary variables in the study participants

\begin{tabular}{|c|c|c|c|c|}
\hline & & ET group & CT group & \\
\hline Variable & & Mean \pm SD & Mean \pm SD & ANOVA interaction \\
\hline Resting $\mathrm{SpO}_{2}(\%)$ & $\mathrm{T}_{0}$ & $91.1 \pm 3.0$ & $93.1 \pm 3.1$ & 0.043 \\
\hline & $\mathrm{T}_{12}$ & $92.1 \pm 3.1$ & $92.1 \pm 3.6^{*}$ & \\
\hline & $\% \Delta$ & $1.1 \pm 3.1$ & $-1.1 \pm 1.8$ & \\
\hline FVC (\% pred) & $\mathrm{T}_{0}$ & $72.9 \pm 22.4$ & $82.2 \pm 25.3$ & 0.237 \\
\hline & $\mathrm{T}_{12}$ & $73.8 \pm 17.2$ & $72.7 \pm 21.8$ & \\
\hline & $\% \Delta$ & $1.2 \pm 24.3$ & $-11.6 \pm 24.3$ & \\
\hline $\mathrm{FEV}_{1}(\%$ pred $)$ & $\mathrm{T}_{0}$ & $43.1 \pm 19.0$ & $57.8 \pm 15.3$ & 0.776 \\
\hline & $\mathrm{T}_{12}$ & $42.6 \pm 19.6$ & $55.8 \pm 15.4$ & \\
\hline & $\% \Delta$ & $-1.2 \pm 12.1$ & $-3.5 \pm 24.4$ & \\
\hline $\mathrm{FEV}_{1} / \mathrm{FVC}(\%)$ & $\mathrm{T}_{0}$ & $45.6 \pm 14.9$ & $54.3 \pm 10.6$ & 0.032 \\
\hline & $\mathrm{T}_{12}$ & $43.0 \pm 11.3$ & $58.6 \pm 10.4^{*}$ & \\
\hline & $\% \Delta$ & $-5.7 \pm 15.4$ & $7.7 \pm 12.2$ & \\
\hline $\mathrm{VO}_{2 \text { peak }}$ & $\mathrm{T}_{0}$ & $17.7 \pm 4.6$ & $15.8 \pm 3.8$ & 0.023 \\
\hline$\left(\mathrm{mL} \mathrm{kg}^{-1} \min ^{-1}\right)$ & $\mathrm{T}_{12}$ & $20.2 \pm 3.6^{*}$ & $15.8 \pm 3.5$ & \\
\hline & $\% \Delta$ & $14.1 \pm 16.9$ & $-0.6 \pm 8.9$ & \\
\hline $\mathrm{W}_{\text {peak }}(\mathrm{W})$ & $\mathrm{T}_{0}$ & $57.2 \pm 27.3$ & $62.3 \pm 29.7$ & 0.010 \\
\hline & $\mathrm{T}_{12}$ & $78.3 \pm 26.5^{* *}$ & $64.1 \pm 32.1$ & \\
\hline & $\% \Delta$ & $36.9 \pm 30.8$ & $2.9 \pm 20.1$ & \\
\hline
\end{tabular}

Abbreviations: $\% \Delta$, percentage change between week 0 and $12 ; \mathrm{CT}$, control; ET, exercise training; SD, standard deviation; $\mathrm{SpO} 2$, peripheral capillary oxygen saturation; $\mathrm{T}_{0}$, week $0 ; \mathrm{T}_{12}$, week $12 ; \mathrm{VO}_{2 \text { peak }}$, peak pulmonary oxygen uptake during cardiopulmonary exercise testing; $\mathrm{W}_{\text {peak }}$, peak work rate during cardiopulmonary exercise testing.

Bold values denote a significat time-by-group interaction $(P<0.05)$.

*Significant differences compared with baseline $(P<0.05)$.

**Significant differences compared with baseline $(P<0.01)$. 


\begin{tabular}{|c|c|c|c|c|}
\hline \multirow[b]{2}{*}{ Variable } & & \multirow{2}{*}{$\frac{\text { ET group }}{\text { Mean } \pm \text { SD }}$} & \multirow{2}{*}{$\frac{\text { CT group }}{\text { Mean } \pm \text { SD }}$} & \multirow[b]{2}{*}{ ANOVA interaction } \\
\hline & & & & \\
\hline \multirow[t]{3}{*}{$\operatorname{MIF}(\mathrm{N})$} & $\mathrm{T}_{0}$ & $1139.5 \pm 532.9$ & $1111.3 \pm 302.6$ & \multirow[t]{3}{*}{0.048} \\
\hline & $\mathrm{T}_{12}$ & $1257.5 \pm 477.4$ & $1000.2 \pm 316.7$ & \\
\hline & $\% \Delta$ & $10.4 \pm 26.7$ & $-10.0 \pm 18.7$ & \\
\hline \multirow{3}{*}{$\begin{array}{l}\text { RFD at } 100 \mathrm{~ms} \\
\left(\mathrm{~N} \mathrm{~s}^{-1}\right)\end{array}$} & $\mathrm{T}_{0}$ & $2371.2 \pm 1741.9$ & $1762.5 \pm 1389.5$ & \multirow[t]{3}{*}{0.009} \\
\hline & $\mathrm{T}_{12}$ & $3917.3 \pm 2789.0^{*}$ & $1623.3 \pm 1650.8$ & \\
\hline & $\% \Delta$ & $65.2 \pm 84.8$ & $-7.9 \pm 30.6$ & \\
\hline \multirow{3}{*}{$\begin{array}{l}\text { RFD at } 200 \mathrm{~ms} \\
\left(\mathrm{~N} \mathrm{~s}^{-1}\right)\end{array}$} & $\mathrm{T}_{0}$ & $2418.7 \pm 1723.4$ & $2048.7 \pm 1142.2$ & \multirow[t]{3}{*}{0.004} \\
\hline & $\mathrm{T}_{12}$ & $3598.2 \pm 1998.4^{*}$ & $1819.2 \pm 1250.8$ & \\
\hline & $\% \Delta$ & $48.8 \pm 54.2$ & $-11.2 \pm 35.1$ & \\
\hline \multirow{3}{*}{$\begin{array}{l}\text { RFD at } 400 \mathrm{~ms} \\
\left(\mathrm{~N} \mathrm{~s}^{-1}\right)\end{array}$} & $\mathrm{T}_{0}$ & $1842.3 \pm 1151.3$ & $1686.3 \pm 774.1$ & \multirow[t]{3}{*}{0.003} \\
\hline & $\mathrm{T}_{12}$ & $2427.0 \pm 1093.5^{*}$ & $1529.9 \pm 716.4$ & \\
\hline & $\% \Delta$ & $31.7 \pm 36.6$ & $-9.3 \pm 21.9$ & \\
\hline \multirow{3}{*}{$\begin{array}{l}\text { Time to } 0.5 \mathrm{BW} \\
\text { (s) }\end{array}$} & $\mathrm{T}_{0}$ & $0.23 \pm 0.16$ & $0.23 \pm 0.11$ & \multirow[t]{3}{*}{0.030} \\
\hline & $\mathrm{T}_{12}$ & $0.14 \pm 0.09$ & $0.26 \pm 0.11$ & \\
\hline & $\% \Delta$ & $-39.3 \pm 28.9$ & $2.5 \pm 10.8$ & \\
\hline \multirow{3}{*}{$\begin{array}{l}\text { 4-m HGS } \\
\left(\mathrm{m} \mathrm{s}^{-1}\right)\end{array}$} & $\mathrm{T}_{0}$ & $1.10 \pm 0.23$ & $1.01 \pm 0.24$ & \multirow[t]{3}{*}{0.030} \\
\hline & $\mathrm{T}_{12}$ & $1.19 \pm 0.17$ & $0.96 \pm 0.23$ & \\
\hline & $\% \Delta$ & $8.2 \pm 15.5$ & $-5.0 \pm 10.9$ & \\
\hline \multirow[t]{3}{*}{ 5-STS time (s) } & $\mathrm{T}_{0}$ & $11.5 \pm 3.3$ & $12.0 \pm 3.3$ & \multirow[t]{3}{*}{0.039} \\
\hline & $\mathrm{T}_{12}$ & $8.7 \pm 2.4 * *$ & $13.8 \pm 8.4$ & \\
\hline & $\% \Delta$ & $-24.3 \pm 15.7$ & $15.8 \pm 51.7$ & \\
\hline \multirow[t]{3}{*}{ SPPB score } & $\mathrm{T}_{0}$ & $10.0 \pm 2.4$ & $9.9 \pm 2.5$ & \multirow[t]{3}{*}{0.109} \\
\hline & $\mathrm{T}_{12}$ & $10.8 \pm 1.5^{*}$ & $10.0 \pm 2.3$ & \\
\hline & $\% \Delta$ & $8.0 \pm 8.0$ & $1.0 \pm 11.1$ & \\
\hline \multirow[t]{3}{*}{ 6-MWD (m) } & $\mathrm{T}_{0}$ & $420.3 \pm 111.6$ & $400.1 \pm 117.5$ & \multirow[t]{3}{*}{0.064} \\
\hline & $\mathrm{T}_{12}$ & $437.1 \pm 85.4$ & $385.0 \pm 135.9$ & \\
\hline & $\% \Delta$ & $4.0 \pm 6.7$ & $-3.8 \pm 10.8$ & \\
\hline
\end{tabular}

T A B L E 3 Effects of exercise training on isometric force variables and physical performance in the study participants

Abbreviations: MIF, maximal isometric force; RFD, rate of force development; BW, body weight; HGS,

habitual gait speed; STS, sit-to-stand; SPPB, short physical performance battery; 6-MWD, 6-min walking distance; ET, exercise training; CT, control; SD, standard deviation; $\mathrm{T}_{0}$, week $0 ; \mathrm{T}_{12}$, week $12 ; \% \Delta$, percentage change between week 0 and 12

Bold values denote a significant time-by-group interaction $(P<0.05)$.

*Significant differences compared with baseline $(P<0.05)$.

$* *$ Significant differences compared with baseline $(P<0.01)$.

CT subjects (only changes in STS time among ET subjects reached statistical significance, $P<0.05$ ). No significant time $\times$ group interaction was observed in the SPPB score, although the ET group improved it by 0.8 pts $(P<0.05)$, with no changes detected in the $\mathrm{CT}$ group $(+0.1 \mathrm{pts} ; P>0.05)$. A trend was shown for the time $\times$ group interaction in the 6-MWD $(P=0.064)$, with no significant changes being detected in either the ET $(+17 \mathrm{~m})$ or the CT $(-15 \mathrm{~m})$ group. A significant time $\times$ group interaction was observed for EQ-VAS $(P<0.05)$, but not for EQ-index values, with ET subjects showing a $20.3 \%$ improvement (from $65.6 \pm 11.0$ to $78.9 \pm 11.7 \mathrm{pts}$ ) and CT subjects a $15.1 \%$ worsening (from
$74.2 \pm 12.2$ to $63.1 \pm 20.5$ pts) in self-reported health status (both $P>0.05$ ).

\section{6 | Systemic oxidative stress}

A significant time $\times$ group interaction was found in protein carbonylation levels $(P<0.05)$ (Figure 5). Protein carbonyls decreased in the ET group $(-26.9 \%$; $P<0.05)$, while no changes were reported among CT subjects $(+25.3 \%$; $P>0.05)$. At baseline, protein carbonylation was associated with $\mathrm{FEV}_{1}(\mathrm{r}=-0.52 ; P=0.006)$ and BODE index $(r=-0.38 ; P=0.049)$. In addition, changes in protein 

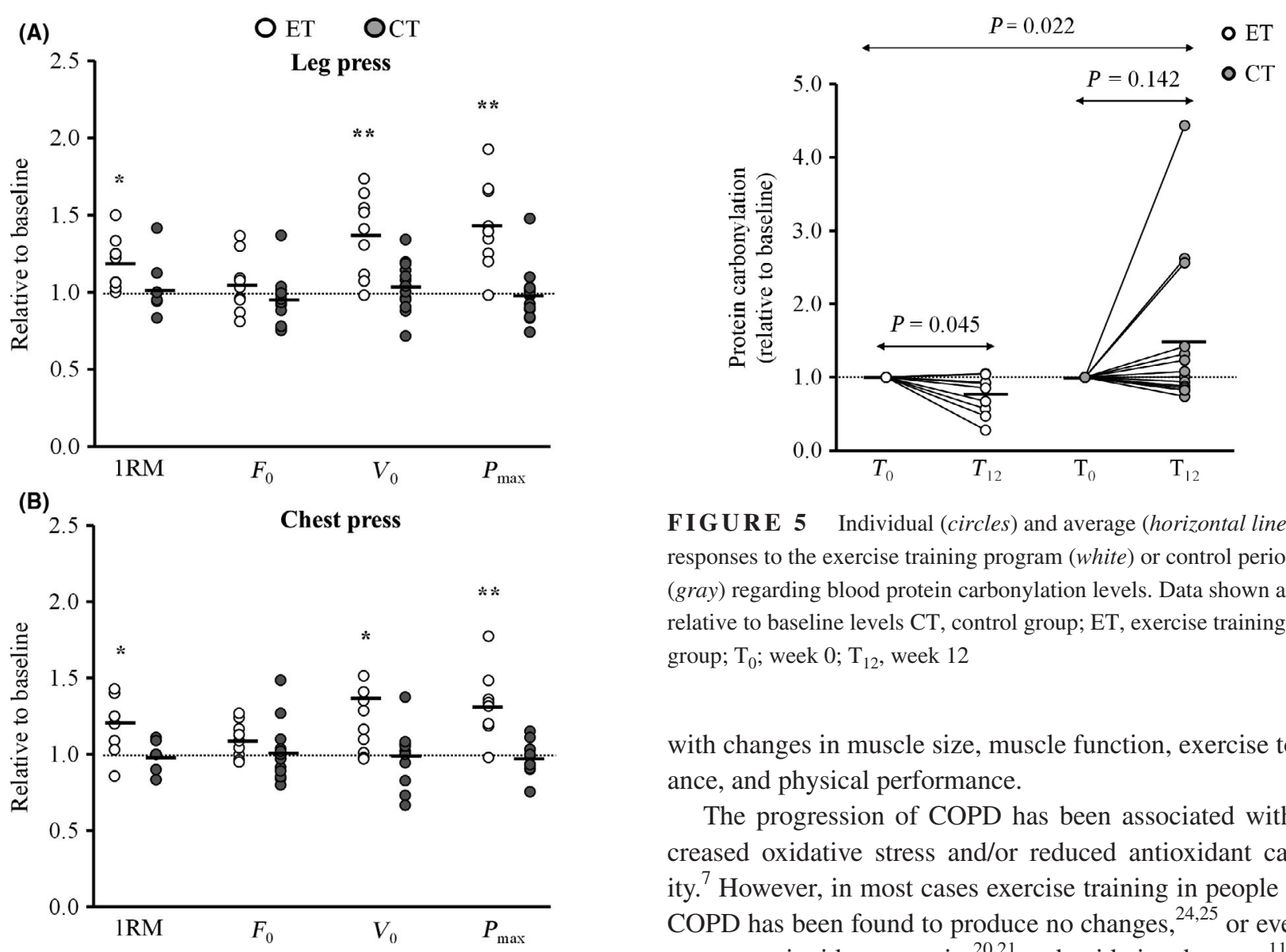

F I G U RE 4 Effects of the exercise training program on leg press (A) and chest press (B) outcomes obtained from the force-velocity relationship of the participants. Individual (circles) and average (horizontal lines) responses to the exercise training program (white) or control period (gray) are shown relative to baseline levels. 1RM, one repetition maximum; CT, control group; ET, exercise training group; $\mathrm{F}_{0}$, force-intercept; $\mathrm{P}_{\max }$, maximal muscle power; $\mathrm{V}_{0}$, velocityintercept. *Significant differences compared with baseline $(P<0.05)$.

${ }^{* *}$ Significant differences compared with baseline $(P<0.01)$

carbonylation over the 12-week period were associated with changes in mid-thigh muscle CSA $(r=-0.44)$, VL muscle thickness $(r=-0.46)$ and pennation angle $(r=-0.57)$, $\mathrm{VO}_{2 \text { peak }}(r=-0.46)$, MVIC $(r=-0.45)$, and STS time $(r=0.60)($ all $P<0.05)$.

\section{DISCUSSION}

The main findings of the present investigation were that a 12week exercise training program combining HIIT and power training in older subjects with COPD produced significant improvements in limb muscle dysfunction, exercise tolerance, systemic oxidative stress, physical performance, and self-reported health status. In addition, changes in systemic oxidative stress were significantly and negatively associated

F I G U R E 5 Individual (circles) and average (horizontal lines) responses to the exercise training program (white) or control period (gray) regarding blood protein carbonylation levels. Data shown as relative to baseline levels $\mathrm{CT}$, control group; ET, exercise training group; $\mathrm{T}_{0}$; week $0 ; \mathrm{T}_{12}$, week 12

with changes in muscle size, muscle function, exercise tolerance, and physical performance.

The progression of COPD has been associated with increased oxidative stress and/or reduced antioxidant capacity. ${ }^{7}$ However, in most cases exercise training in people with COPD has been found to produce no changes, ${ }^{24,25}$ or even to worsen antioxidant capacity ${ }^{20,21}$ and oxidative damage. ${ }^{11,22,23}$ By contrast, in the present study we found a positive effect of concurrent exercise training on blood protein carbonylation levels in older people with COPD. In this line, Rodriguez et $\mathrm{al}^{29}$ observed a positive effect of exercise training on protein nitration levels in patients with COPD, and although a significant effect in blood or muscle protein carbonylation levels was not observed, a reduction in protein carbonyls can be regarded in their COPD subjects with the highest levels of oxidative stress at baseline. Moreover, Ryrs $\varnothing$ et al ${ }^{30}$ recently noted an improved muscle antioxidant capacity in COPD subjects after completing an exercise program. Discrepancies among studies may be due to differences in exercise prescription. A single bout of exercise exceeding a certain intensity or duration induces an exaggerated production of RONS and oxidative damage in people with COPD. ${ }^{31,32}$ Hence, the exercise type and dose must be carefully managed when it comes to sedentary older people with COPD. Our proposed exercise program and the ones proposed by Rodriguez et $\mathrm{al}^{29}$ and Ryrs $\varnothing$ et $\mathrm{al}^{30}$ coincide in that the exercise dose was less strenuous compared with the studies showing no changes or an increased oxidative stress among COPD participants. ${ }^{11,20-23,25}$ Actually, increased oxidative stress in those studies might be a consequence of overtraining due to the inability of the subjects to adapt to the received exercise dose. ${ }^{33}$ In addition, our exercise program differed from those found in other studies with COPD patients in that it integrated power training as 
a component. Notably, power training induces lower cardiovascular loading ${ }^{17}$ and maintains higher muscle oxygenation levels ${ }^{18}$ during exercise compared with traditional strength training, which could also contribute to the positive redox response observed in the present study.

Studies with animals suggest that exercise training can increase the activity of several antioxidant enzymes such as superoxide dismutase (SOD), catalase, or glutathione peroxidase (GPX). ${ }^{34}$ Ryrs $\varnothing$ et al ${ }^{30}$ found an increase in SOD2 protein levels among COPD participants, while Rodriguez et $\mathrm{al}^{29}$ observed changes in protein carbonylation to be negatively associated with training-induced changes in catalase. Oxidative stress can also induce the formation of heat shock proteins (HSPs) as an important component of the cellular protective response against oxidative damage. ${ }^{34}$ In addition, exercise-induced mitochondria remodeling might also positively affect oxidative stress, since the mitochondrial respiratory chain is one of the principal sources of excessive RONS generation in the pathophysiological context of COPD. ${ }^{4} \mathrm{~A}$ trend for increased UCP3 protein levels (a protein suggested to protect mitochondria against lipid peroxide-induced damage) was observed among patients with COPD after participating in a concurrent endurance and resistance training program. ${ }^{25}$ The identification of the potential mechanisms leading to the improved systemic oxidative stress observed in our older subjects with COPD warrants further investigation.

On the other hand, we found changes in systemic oxidative stress to be significantly and negatively associated with changes in muscle size and strength, exercise capacity, and physical performance. Rodriguez et $\mathrm{al}^{29}$ also found changes in protein carbonyls to be negatively associated with changes in $\mathrm{VO}_{2 \text { peak }}$ among patients with COPD. The reaction of RONS with proteins results in the modification of protein structures, the formation of protein aggregates, and/ or the cleavage of peptide bonds, making proteins dysfunctional and more susceptible to proteinases or complete protein unfolding. ${ }^{35}$ Proteins affected by oxidative stress have been reported to be involved in glycolysis, energy distribution, carbon dioxide hydration, muscle oxygen transfer, DNA repair, and muscle contractile function. ${ }^{11}$ Thus, excessive RONS generation might blunt exercise-induced adaptations through its influence on different proteolytic systems that can act simultaneously. ${ }^{36}$ Increased levels of muscle-specific E3 ligases MURF-1 and atrogin-1 were found in patients with COPD, and oxidative stress was associated with total levels of protein ubiquitination. ${ }^{9}$ Levels of activated transcription factors such as NF- $\kappa \beta$ p65, I- $\kappa \beta$-alpha, FoxO-1, and FoxO-3 were increased in cachectic COPD patients compared with healthy counterparts. ${ }^{9}$ Oxidative stress is associated with increased growth differentiation factor- 15 in patients with COPD, which is known to contribute to the loss of muscle mass in COPD. ${ }^{37}$ It is also clear that oxidative stress can contribute to the rate of disuse muscle atrophy by promoting the activation of calpain and/or caspase- $3 .{ }^{38}$ Finally, oxidative stress can directly affect muscle contractility by a reduction in the activity of the sarcoplasmic reticulum and the disruption of excitation-contraction coupling, ${ }^{39}$ or by leading to enzymatic and muscle metabolic dysfunction. ${ }^{40}$ These mechanisms would explain the significant association between changes in oxidative stress and changes in muscle size and function, exercise tolerance, and physical performance noted in our subjects.

The combination of HIIT and power training provided significant changes in aerobic capacity and muscle function. Our reported exercise-induced adaptations in $\mathrm{VO}_{2 \text { peak }}$ might be considered similar to those observed in previous studies using HIIT alone $e^{20,21}$ or a combination of endurance and strength training, ${ }^{31}$ while the adaptations in $\mathrm{W}_{\text {peak }}$ seemed to be greater in our study compared with previous investigations, ${ }^{20,23,31,41}$ perhaps because of the effects derived from power training. Adaptations in $\mathrm{VO}_{2 \text { peak }}$ might have been promoted by an increased ability to supply oxygen to the working muscles, given the local reserve capacity to consume oxygen that has been reported in COPD subjects. ${ }^{42}$ By contrast, no effects were observed in lung function variables, which are more likely to improve with the inclusion of inspiratory muscle training. ${ }^{43}$ Of note, the non-exercising group improved FEV1/FVC values, but it was actually due to a non-significant albeit substantial decline in FVC values $(-12 \%)$, while the trained group preserved FVC during the 12-week period.

Substantial positive changes were observed in our exercising subjects in terms of explosive force capacity and muscle power. Interestingly, we observed a positive effect of the exercise program on the time needed to reach a target level of force relative to the individuals' body mass, which may have important implications in fall prevention. In addition, our exercising subjects showed substantially higher lower-limb muscle power adaptations compared with those previously reported in COPD participants (51\% vs $19 \%$ ). ${ }^{44,45}$ The fact that our subjects were specifically instructed to perform each repetition as fast and strong as possible with the load that elicited maximal power output might be behind these positive results. In terms of muscle hypertrophy, we observed a 5\% increase in mid-thigh muscle CSA and an $11 \%$ increase in VL muscle thickness among the trained subjects. Similar increments have been reported in the literature in resistancetrained COPD patients $(4 \%-11 \%){ }^{23,45-47}$ Although muscle excitation levels were not registered in our subjects, the disparity between adaptations in muscle size and muscle function, along with the greater adaptations observed in RFD at $100 \mathrm{~ms}$ compared with $400 \mathrm{~ms}$, suggests that the improvement in muscle power might have been mostly driven by neural adaptations. ${ }^{48}$

One of the novelties of our investigation was the evaluation of muscle architecture in older subjects with COPD. It has been previously reported that patients with COPD show a 
loss of normal sarcomere architecture (ie, microscopic architecture) related to a loss of thick filaments or reduced protein content of contractile myosin. ${ }^{9}$ Our exercise intervention elicited positive adaptations in VL pennation angle (ie, macroscopic architecture), but not in VL fascicle length. This might be due to the fact that while a maximal volitional effort was performed during the concentric portion of the leg press exercise, no specific instructions were given for the eccentric portion of the exercise. Thus, we noted that most of the subjects preferred to perform the eccentric phase quickly, which reduced the fascicle force levels when lowering the load. In this regard, the evidence suggests that concentric loading leads to an increased pennation angle (an indirect indicator of the addition of sarcomeres in parallel) and that eccentric loading leads to increases in fascicle length (by the addition of sarcomeres in series). ${ }^{49}$ Thus, perhaps controlling the eccentric phase (2-3 s) and the transition to the concentric phase of the exercise might promote greater adaptations in fascicle length.

Finally, positive changes were observed in the exercising COPD participants in terms of physical performance. Importantly, changes in gait speed, STS time, and SPPB score among the COPD subjects participating in the exercise program reached the thresholds for a minimal important difference ( $\geq 0.05 \mathrm{~m} \mathrm{~s}^{-1}, \geq 2.5 \mathrm{~s}$ and $\geq 0.8 \mathrm{pts}$, respectively). By contrast, our subjects did not experience relevant changes in 6-MWD (ie, $<25 \mathrm{~m}$ ), although it should be pointed out that the opportunity cost of not exercising vs exercising (ie, the difference between the change experimented by the ET and CT groups) was $32 \mathrm{~m}$, which is above the threshold for a relevant change in 6-MWD. In any case, walking-based exercise programs are considered to provide greater benefits in walking endurance. ${ }^{50}$ Finally, the abovementioned positive changes derived from the participation of the older subjects with COPD in the exercise program probably led to the observed improvement in self-reported health status (ie, EQVAS). Notably, the change in the EQ-VAS was higher than the minimum important difference required in patients with COPD (>6.9 pts).

One of the limitations of the present investigation was that systemic oxidative stress was evaluated through protein carbonylation levels, while other additional markers such as malondialdehyde or 8-hydroxydeoxyguanosine might also have been of interest. However, protein carbonylation is the most abundant by-product of oxidative-induced protein damage, ${ }^{5}$ which has become the most generally used method for estimating oxidative stress-mediated protein oxidation, ${ }^{51}$ and the procedure used in this investigation has shown the best sensitivity and specificity. ${ }^{52}$ Moreover, protein carbonylation levels are of great relevance for people with COPD due to their association with disease severity, limb muscle dysfunction, and exercise tolerance. ${ }^{7-11}$ On the other hand, we observed a greater number of dropouts in the ET group compared with the CT group. It has been reported in the literature that $10 \%-32 \%$ of the COPD subjects participating in a pulmonary rehabilitation program usually dropout. ${ }^{53}$ The main barriers that COPD subjects usually report are difficulties with transportation, mobility, distance, and location of programs, which is more likely to affect the subjects participating in the exercise program. In addition, patients with COPD can experience disease exacerbations that impede them from attending the exercise sessions. In this sense, the implementation of home-based exercise training programs or the use of NMES at home might be indicated in order to at least minimize the loss of exercise-induced adaptations between supervised training sessions in patients with COPD unable to travel to the exercise facilities.

In conclusion, an exercise training program combining HIIT and power training partially reverted limb muscle dysfunction, improved exercise tolerance and physical performance in older people with COPD and positively influenced their self-reported health status. Importantly, the exercising COPD participants experienced a decrease in systemic oxidative stress that in turn was associated with changes in muscle size and strength, aerobic capacity, and physical performance.

\section{5 | PERSPECTIVES}

The identification of potential therapies targeting oxidative stress in people with COPD may be beneficial to prevent disease progression and other COPD-related consequences. A recent study demonstrated the antioxidant benefits derived from exercise participation in people with $\mathrm{COPD},{ }^{30}$ but it remained unknown whether the absolute level of oxidative stress remained unchanged. Our findings add novel evidence on the benefits derived from concurrent exercise training on systemic oxidative stress in older people with COPD. Oxidative stress may play a role as a regulator of exerciseinduced structural and functional adaptations in skeletal muscles of patients with COPD. In this regard, the effects of exercise training on oxidative stress in patients with COPD might be tightly regulated by exercise dosage, and thus, exercise programs should be carefully prescribed in sedentary older people with COPD in order to maximize their benefits. Future studies should be conducted in order to assess the dose-response relationship between exercise training and oxidative stress in subjects with COPD, as well as the effect of adding power training to conventional pulmonary rehabilitation programs.

\section{ACKNOWLEDGEMENTS}

We thank all the participants for their collaboration and involvement in the study. 
CONFLICTS OF INTEREST

The authors declare no conflicts of interest.

\section{ORCID}

Julian Alcazar (iD) https://orcid.org/0000-0002-1090-5482

Luis M. Alegre (D) https://orcid.org/0000-0002-4502-9275

Amelia Guadalupe-Grau (D) https://orcid.

org/0000-0003-2573-4572

\section{REFERENCES}

1. Vogelmeier CF, Criner GJ, Martinez FJ, et al. Global strategy for the diagnosis, management, and prevention of chronic obstructive lung disease 2017 report: GOLD executive summary. Arch Bronconeumol. 2017;53(3):128-149.

2. Maltais F, Decramer M, Casaburi R, et al. An official American Thoracic Society/European Respiratory Society statement: update on limb muscle dysfunction in chronic obstructive pulmonary disease. Am J Respir Crit Care Med. 2014;189(9):e15-62.

3. Barreiro E, Fermoselle C, Mateu-Jimenez M, et al. Oxidative stress and inflammation in the normal airways and blood of patients with lung cancer and COPD. Free Radic Biol Med. 2013;65:859-871.

4. Couillard A, Prefaut C. From muscle disuse to myopathy in COPD: potential contribution of oxidative stress. Eur Respir J. 2005;26(4):703-719.

5. Zinellu E, Zinellu A, Fois AG, Carru C, Pirina P. Circulating biomarkers of oxidative stress in chronic obstructive pulmonary disease: a systematic review. Respir Res. 2016;17(1):150.

6. Kirkham P. Oxidative stress and macrophage function: a failure to resolve the inflammatory response. Biochem Soc Trans. 2007;35(Pt 2):284-287.

7. Rahman I, van Schadewijk A, Crowther A, et al. 4-Hydroxy-2-nonenal, a specific lipid peroxidation product, is elevated in lungs of patients with chronic obstructive pulmonary disease. Am J Respir Crit Care Med. 2002;166(4):490-495.

8. Van Helvoort HA, Heijdra YF, Thijs HM, Vina J, Wanten GJ, Dekhuijzen PN. Exercise-induced systemic effects in muscle-wasted patients with COPD. Med Sci Sports Exerc. 2006;38(9):1543-1552.

9. Puig-Vilanova E, Rodriguez DA, Lloreta J, et al. Oxidative stress, redox signaling pathways, and autophagy in cachectic muscles of male patients with advanced COPD and lung cancer. Free Radic Biol Med. 2015;79:91-108.

10. Barreiro E, Schols A, Polkey MI, et al. Cytokine profile in quadriceps muscles of patients with severe COPD. Thorax. 2008;63(2):100-107.

11. Barreiro E, Rabinovich R, Marin-Corral J, Barbera JA, Gea J, Roca J. Chronic endurance exercise induces quadriceps nitrosative stress in patients with severe COPD. Thorax. 2009;64(1):13-19.

12. McCarthy B, Casey D, Devane D, Murphy K, Murphy E, Lacasse Y. Pulmonary rehabilitation for chronic obstructive pulmonary disease. Cochrane Database Syst Rev. 2015;(2);Cd003793. https:// www.ncbi.nlm.nih.gov/pubmed/25705944

13. Alcazar J, Rodriguez-Lopez C, Alfaro-Acha A, Alegre LM, Ara I. Exercise prescription in patients with chronic obstructive pulmonary disease. Eur J Hum Mov. 2018; 41: 73 - 102.
14. Zainuldin R, Mackey MG, Alison JA. Optimal intensity and type of leg exercise training for people with chronic obstructive pulmonary disease. Cochrane Database Syst Rev. 2011;(11):Cd008008. https://www.ncbi.nlm.nih.gov/pubmed/22071841

15. Liao W-H, Chen J-W, Chen X, et al. Impact of resistance training in subjects with COPD: a systematic review and meta-analysis. Respir Care. 2015;60(8):1130-1145.

16. Byrne C, Faure C, Keene DJ, Lamb SE. Ageing, muscle power and physical function: a systematic review and implications for pragmatic training interventions. Sports Med (Auckland, NZ). 2016;46(9):1311-1332.

17. Lamotte M, Fleury F, Pirard M, Jamon A, van de Borne P. Acute cardiovascular response to resistance training during cardiac rehabilitation: effect of repetition speed and rest periods. Eur $J$ Cardiovasc Prev Rehabil. 2010;17(3):329-336.

18. Tanimoto M, Ishii N. Effects of low-intensity resistance exercise with slow movement and tonic force generation on muscular function in young men. J Appl Physiol. 2006;100(4):1150-1157.

19. Navarro-Cruz R, Alcazar J, Rodriguez-Lopez C, et al. The effect of the stretch-shortening cycle in the force-velocity relationship and its association with physical function in older adults with COPD. Front Physiol. 2019;10:316.

20. Rabinovich RA, Ardite E, Troosters T, et al. Reduced muscle redox capacity after endurance training in patients with chronic obstructive pulmonary disease. Am J Respir Crit Care Med. 2001;164(7):1114-1118.

21. Rabinovich RA, Ardite E, Mayer AM, et al. Training depletes muscle glutathione in patients with chronic obstructive pulmonary disease and low body mass index. Respiration. 2006;73(6):757-761.

22. Pinho RA, Chiesa D, Mezzomo KM, et al. Oxidative stress in chronic obstructive pulmonary disease patients submitted to a rehabilitation program. Respir Med. 2007;101(8):1830-1835.

23. Vogiatzis I, Simoes D, Stratakos G, et al. Effect of pulmonary rehabilitation on muscle remodelling in cachectic patients with COPD. Eur Respir J. 2010;36(2):301-310.

24. Chavoshan B, Fournier M, Lewis MI, et al. Testosterone and resistance training effects on muscle nitric oxide synthase isoforms in COPD men. Respir Med. 2012;106(2):269-275.

25. Gosker HR, Schrauwen P, Broekhuizen R, et al. Exercise training restores uncoupling protein-3 content in limb muscles of patients with chronic obstructive pulmonary disease. Am J Physiol Endocrinol Metab. 2006;290(5):E976-E981.

26. Celli BR, Cote CG, Marin JM, et al. The body-mass index, airflow obstruction, dyspnea, and exercise capacity index in chronic obstructive pulmonary disease. N Engl J Med. 2004;350(10):1005-1012.

27. Alegre LM, Aguado X, Rojas-Martin D, Martin-Garcia M, Ara I, Csapo R. Load-controlled moderate and high-intensity resistance training programs provoke similar strength gains in young women. Muscle Nerve. 2015;51(1):92-101.

28. Alcazar J, Rodriguez-Lopez C, Ara I, et al. The force-velocity relationship in older people: reliability and validity of a systematic procedure. Int J Sports Med. 2017;38(14):1097-1104.

29. Rodriguez DA, Kalko S, Puig-Vilanova E, et al. Muscle and blood redox status after exercise training in severe COPD patients. Free Radic Biol Med. 2012;52(1):88-94.

30. Ryrs $\varnothing$ CK, Thaning P, Siebenmann C, et al. Effect of endurance versus resistance training on local muscle and systemic inflammation and oxidative stress in COPD. Scand J Med Sci Sports. 2018;28(11):2339-2348. 
31. Mercken EM, Hageman GJ, Schols AM, Akkermans MA, Bast A, Wouters EF. Rehabilitation decreases exercise-induced oxidative stress in chronic obstructive pulmonary disease. Am J Respir Crit Care Med. 2005;172(8):994-1001.

32. Couillard A, Maltais F, Saey D, et al. Exercise-induced quadriceps oxidative stress and peripheral muscle dysfunction in patients with chronic obstructive pulmonary disease. Am J Respir Crit Care Med. 2003;167(12):1664-1669.

33. Palazzetti S, Richard M-J, Favier A, Margaritis I. Overloaded training increases exercise-induced oxidative stress and damage. Can J Appl Physiol. 2003;28(4):588-604.

34. Khassaf M, Child RB, McArdle A, Brodie DA, Esanu C, Jackson MJ. Time course of responses of human skeletal muscle to oxidative stress induced by nondamaging exercise. J App Physiol. 2001;90(3):1031-1035.

35. Dean RT, Fu S, Stocker R, Davies MJ. Biochemistry and pathology of radical-mediated protein oxidation. Biochem J. 1997;324(Pt 1):1-18.

36. Hussain SN, Sandri M. Role of autophagy in COPD skeletal muscle dysfunction. J Appl Physiol (Bethesda, Md : 1985). 2013;114(9):1273-1281.

37. Patel MS, Lee J, Baz M, et al. Growth differentiation factor- 15 is associated with muscle mass in chronic obstructive pulmonary disease and promotes muscle wasting in vivo. J Cachexia Sarcopenia Muscle. 2016;7(4):436-448.

38. Powers SK, Jackson MJ. Exercise-induced oxidative stress: cellular mechanisms and impact on muscle force production. Physiol Rev. 2008;88(4):1243-1276.

39. Qaisar R, Bhaskaran S, Premkumar P, et al. Oxidative stress-induced dysregulation of excitation-contraction coupling contributes to muscle weakness. J Cachexia Sarcopenia Muscle. 2018;9:1003-1017.

40. Andersson U, Leighton B, Young ME, Blomstrand E, Newsholme EA. Inactivation of aconitase and oxoglutarate dehydrogenase in skeletal muscle in vitro by superoxide anions and/or nitric oxide. Biochem Biophys Res Commun. 1998;249(2):512-516.

41. Bolton CE, Broekhuizen R, Ionescu AA, et al. Cellular protein breakdown and systemic inflammation are unaffected by pulmonary rehabilitation in COPD. Thorax. 2007;62(2):109-114.

42. Helgerud J, Bjørgen S, Karlsen T, et al. Hyperoxic interval training in chronic obstructive pulmonary disease patients with oxygen desaturation at peak exercise. Scand J Med Sci Sports. 2010;20(1):e1 70-e176.

43. Beaumont M, Forget P, Couturaud F, Reychler G. Effects of inspiratory muscle training in COPD patients: a systematic review and meta-analysis. Clin Respir J. 2018;12(7):2178-2188.

44. Zambom-Ferraresi F, Cebollero P, Gorostiaga EM, et al. Effects of combined resistance and endurance training versus resistance training alone on strength, exercise capacity, and quality of life in patients with COPD. J Cardiopulm Rehabil Prev. 2015;35(6):446-453.
45. Kongsgaard M, Backer V, Jorgensen K, Kjaer M, Beyer N. Heavy resistance training increases muscle size, strength and physical function in elderly male COPD-patients-a pilot study. Respir Med. 2004;98(10):1000-1007.

46. Greulich T, Kehr K, Nell C, et al. A randomized clinical trial to assess the influence of a three months training program (gym-based individualized vs. calisthenics-based non-invidualized) in COPDpatients. Respir Res. 2014;15:36.

47. McKeough ZJ, Alison JA, Bye P, et al. Exercise capacity and quadriceps muscle metabolism following training in subjects with COPD. Respir Med. 2006;100(10):1817-1825.

48. Maffiuletti NA, Aagaard P, Blazevich AJ, Folland J, Tillin N, Duchateau J. Rate of force development: physiological and methodological considerations. Eur J Appl Physiol. 2016;116(6):10911116. https://doi.org/10.1007/s00421-016-3346-6.

49. Franchi MV, Atherton PJ, Reeves ND, et al. Architectural, functional and molecular responses to concentric and eccentric loading in human skeletal muscle. Acta Physiol (Oxford, England). 2014;210(3):642-654.

50. Leung RW, Alison JA, McKeough ZJ, Peters MJ. Ground walk training improves functional exercise capacity more than cycle training in people with chronic obstructive pulmonary disease (COPD): a randomised trial. J Physiother. 2010;56(2):105-112.

51. Stadtman ER. Protein oxidation and aging. Free Radic Res. 2006;40(12):1250-1258.

52. Weber D, Davies MJ, Grune T. Determination of protein carbonyls in plasma, cell extracts, tissue homogenates, isolated proteins: Focus on sample preparation and derivatization conditions. Redox Biol. 2015;5:367-380.

53. Keating A, Lee A, Holland AE. What prevents people with chronic obstructive pulmonary disease from attending pulmonary rehabilitation? A systematic review. Chron Respir Dis. 2011;8(2):89-99.

\section{SUPPORTING INFORMATION}

Additional supporting information may be found online in the Supporting Information section at the end of the article.

How to cite this article: Alcazar J, Losa-Reyna J, Rodriguez-Lopez C, et al. Effects of concurrent exercise training on muscle dysfunction and systemic oxidative stress in older people with COPD. 